\title{
Competitividad portuaria en el Pacífico mexicano, con especial referencia a Ensenada, Baja California
}

\section{Port competitiveness in the Mexican Pacific Ocean, with special reference to Ensenada, Baja California} Carlos Israel Vázquez León* y Wilfrido Ruiz Ochoa**

Resumen

Este trabajo tiene como objetivo delimitar y comparar los factores determinantes de la competitividad portuaria de los tres principales puertos de la costa del Océano Pacífico mexicano: Lázaro Cárdenas en Michoacán, Ensenada en Baja California, y Manzanillo en Colima. Tal comparación se realiza mediante el Proceso Analítico Jerárquico, en conjunto con el modelo de Fortalezas, Oportunidades, Debilidades y Amenazas. Se encuentran diferencias notables entre los puertos, tanto en lo referente a las condiciones de infraestructura para la competitividad, como en relación con sus estrategias comerciales. Los resultados colocan al puerto de Ensenada en clara desventaja, lo cual compromete su potencial frente a las oportunidades que brinda la Cuenca del Pacífico y el Corredor Marítimo Este-Oeste del Hemisferio Norte.

Palabras clave: competitividad portuaria, proceso analítico jerárquico.

\section{Abstract}

This work aims, identify and compare the determinants of port competitiveness of the three major ports on the Mexican Pacific coast: Lázaro Cárdenas in Michoacán; Ensenada in Baja California; and Manzanillo in Colima. Such comparison was performed using the Analytic Hierarchy Process along with the model of Strengths, Weaknesses, Opportunities and Threats. Notable differences were found among these ports, both in terms of the infrastructure for competitiveness, as well as their business strategies. The competitive positioning observed from this analysis, shows that the Port of Ensenada is located at a significant disadvantage, which compromises their potential to exploit opportunities offered by Pacific Rim and the East-West Maritime Corridor of the Northern Hemisphere.

Keywords: port competitiveness, analytical hierarchical process.

Recibido el 29 de julio de 2013.

Aprobado el 21 de julio de 2014.

* Profesor-investigador de El Colegio de la Frontera Norte. Departamento de Estudios Urbanos y del Medio Ambiente. Correo electrónico: cvazquez@colef.mx

** Profesor-investigador de El Colegio de la Frontera Norte. Departamento de Estudios Económicos. Correo electrónico: wruiz@colef.mx 


\section{Introducción y objetivos}

La Secretaría de Comunicaciones y Transportes (SCT) estima que para el año 2030 el movimiento de carga vía marítima aumentará de manera considerable, a tal grado que la contribución del transporte marítimo al total de la carga transportada en el país pasará de 31\% en 2009 a casi 38 puntos porcentuales en el año 2030, lo cual obligará a ampliar la actual capacidad instalada del sistema portuario nacional (sCT, 2008). En este escenario, el litoral del Pacífico participará cada vez con una mayor proporción de la carga contenerizada que se moviliza en el país, debido a su gran potencial y ubicación sobre las rutas troncales de Asia y América del Norte. Esta tendencia puede observarse con claridad en la actualidad: en 2012 los puertos del Pacífico participaban con $70 \%$ de la movilidad de carga, lo cual representa prácticamente el doble respecto de la de 2000 (cuadro 1).

Desafortunadamente, el potencial del Pacífico se enfrenta a una infraestructura portuaria desigual. De los 56 puertos y terminales con los que cuenta, solamente 16 son puertos de altura, ${ }^{1}$ y de éstos, sólo ocho participan

\section{Cuadro 1. Comportamiento sexenal de la participación en la carga contenerizada por litoral y puerto, 2000-2012 (porcentaje de TEU's movilizados)}

\begin{tabular}{|l|c|c|c|}
\hline \multicolumn{1}{|c|}{ Litoral y Puerto / Años } & 2000 & 2006 & 2012 \\
\hline Pacífico & 36.28 & 58.44 & 70.15 \\
\hline & 63.72 & 41.56 & 29.85 \\
\hline Golfo y Caribe & & & \\
\hline Composición del Pacífico & & & \\
\hline Manzanillo, Colima & 89.4 & 79.89 & 58.21 \\
\hline Lázaro Cárdenas, Michoacán & 0.16 & 10.27 & 36.32 \\
\hline Mazatlán, Sinaloa & 3.52 & 1.93 & 1.15 \\
\hline Salina Cruz, Oaxaca & 1.12 & - & 0 \\
\hline
\end{tabular}

Fuente: Elaboración propia a partir de Anuarios Estadísticos de la SCT (2001, 2006 y 2012).

${ }^{1}$ La diferencia entre un puerto de altura y uno de cabotaje radica en que mientras el primero se encuentra en condiciones de recibir embarcaciones de gran calado, así como de enviar y recibir carga internacional, los de cabotaje regularmente reciben carga y pasajeros entre puertos nacionales, con limitada o nula cobertura internacional. 
de la captación de carga contenerizada. Para mayor claridad, en la figura 1 se muestran los principales puertos del país que son coordinados por Administraciones Portuarias Integrales.

En el Pacífico destacan los de Lázaro Cárdenas y Manzanillo, por ser los únicos que funcionan como puertos $h u b$ de influencia regional (Martner, 2010), ${ }^{2}$ lo cual justifica su inclusión en este estudio. Tan sólo el corredor Manzanillo-Distrito Federal cuenta con adaptaciones tecnológicas y servicios regulares de estiba sencilla y doble estiba, que le han permitido participar con la mayoría de la carga contenerizada movilizada por los puertos de el Pacífico, ascendiendo la misma a casi 60\% en 2012. Quedando de esta forma relegados el resto de los puertos de la región, a excepción del de Lázaro Cárdenas que moviliza 36\% de los contenedores movilizados en el Pacífico (cuadro 1).

Figura 1. Localización de los principales puertos del Pacífico y el Golfo de México

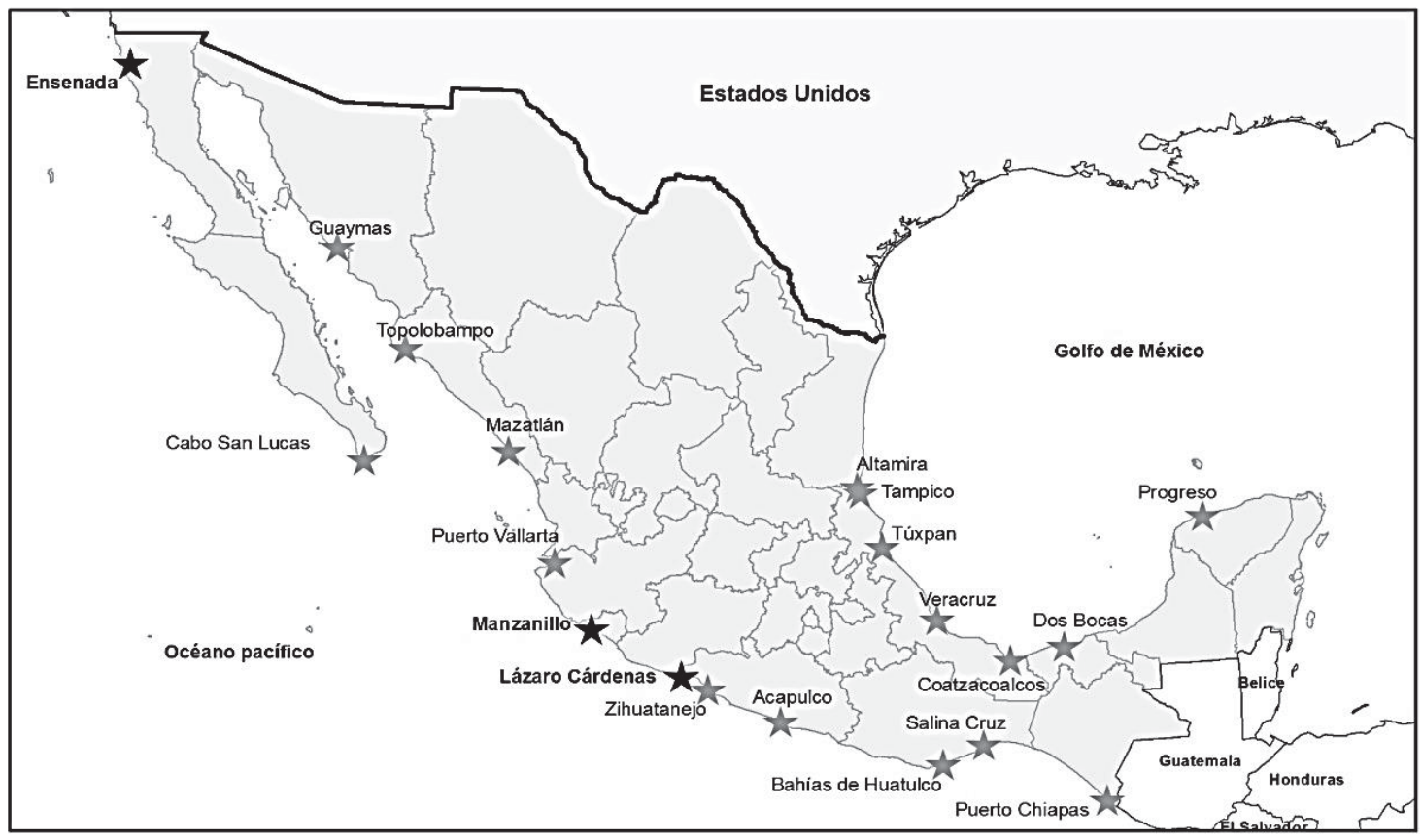

Fuente: Adaptación a partir del mapa utilizado por la Dirección de Puertos y Marina. Ver sст, 2013.

${ }^{2}$ Un puerto hub se caracteriza por concentrar gran cantidad de bienes, que después son distribuidos por embarcaciones de corto recorrido. Habiendo hub de influencia local, regional y global. 
Entre los puertos grandes del Pacífico, el de Lázaro Cárdenas ha sido el más dinámico. Durante el periodo 2000-2012, la carga contenerizada que movilizó este puerto creció tres veces en promedio por año. Muy por encima del de Manzanillo, que alcanzó en el mismo periodo un tasa de crecimiento promedio de $15 \%$. Como explicación, Martner y colaboradores (2010) señalan que a partir de 2008 el puerto michoacano empezó a repuntar gracias al desarrollo multimodal de trenes a doble estiba que lo vinculan con el centro del país y con el Centro-Este de Estados Unidos a través de la frontera de Nuevo Laredo, en el estado de Tamaulipas.

El puerto de Ensenada, por su parte, aunque solamente participa con $4 \%$ de la carga contenerizada del Pacífico, es el mejor ubicado en virtud de su posición en la Cuenca del Pacífico, su cercanía con los hub portuarios de Asia y la Costa Oeste de Estados Unidos, y por encontrarse dentro del Corredor Marítimo Este-Oeste del Hemisferio Norte. Sin embargo, enfrenta obstáculos naturales para conectarse por tierra mediante ferrocarril, así como para ampliar su zona de influencia debido a su limitada reserva territorial. Por ello, se considera que en un futuro su vocación podría ser la de puerto de altura especializado, con potencial para elevar su actividad hasta ciertos umbrales si se logra desarrollar mayor infraestructura complementaria para el mismo. Sobre esto último, puede mencionarse que durante 2000-2006 fue uno de los puertos que mayor crecimiento observó en cuanto a movilidad de carga, gracias al aprovechamiento del auge económico internacional de esos años y a la saturación temporal del puerto de Long Beach (cuadro 2).

En este contexto, se avizora que en las próximas décadas se intensificará la competencia entre aquellos puertos del Pacífico que comparten parte de los mismos ámbitos de influencia, y que se dedican al manejo de carga contenerizada procedente del país y de diversas naciones con las que México mantiene acuerdos comerciales. Requiriéndose entonces un análisis de la competitividad de los puertos mexicanos con el fin de revisar las tendencias en cuanto a su posicionamiento en el entramado nacional y global de los flujos comerciales, y de identificar de mejor manera sus nichos de oportunidad.

Precisamente, este artículo plantea como objetivo central delimitar y analizar aquellos factores que influyen sobre el grado de competitividad de un puerto, acotando este análisis a los determinantes comerciales y a los 


\section{Cuadro 2. Tasa de crecimiento promedio anual del movimiento de carga contenerizada, por litoral y puertos del Pacífico (Porcentajes)}

\begin{tabular}{|l|c|c|c|}
\hline \multicolumn{1}{|c|}{ Litoral y Puerto / Periodo } & $2000-2006$ & $2006-2012$ & $2000-2012$ \\
\hline Pacífico & 22.63 & 18.69 & 18.69 \\
\hline El Sauzal, Baja California & -42.16 & -42.12 & -37.32 \\
\hline Ensenada, Baja California & 38.23 & 11.45 & 20.4 \\
\hline Guaymas, Sonora & -41.08 & 8977.97 & 5216.61 \\
\hline Lázaro Cárdenas, Michoacán & 632.34 & 40.15 & 337.82 \\
\hline Manzanillo, Colima & 20.53 & 14.36 & 15.04 \\
\hline Mazatlán, Sinaloa & 14.22 & 16.85 & 10.98 \\
\hline Salina Cruz, Oaxaca & 51.24 & 61.69 & 69.94 \\
\hline Golfo y Caribe & 4.86 & 5.55 & 5.04 \\
\hline Todos los puertos & 12.77 & 13.29 & 12.02 \\
\hline
\end{tabular}

Fuente: Elaboración propia a partir de los Anuarios Estadísticos de la SCT (2001, 2006 y 2012).

relacionados con las características físicas o de infraestructura de los tres principales puertos de la costa del océano Pacífico mexicano, que son los de Lázaro Cárdenas en Michoacán, Ensenada en Baja California, y Manzanillo en Colima.

Para tal fin, en este trabajo se utiliza un Proceso Analítico Jerárquico —AHP, por sus siglas en inglés-, que valora la competitividad portuaria considerando factores infraestructurales y comerciales que la condicionan. Ésta es una metodología similar a la seguida por Le-Griffin y Murphy (2006), quienes comparan los puertos de Los Ángeles y Long Beach en California con otros grandes puertos a escala mundial. Además de considerar las características físicas portuarias que se retoman de dichos autores, en el presente estudio incluimos determinantes de índole comercial, para valorar la competitividad de los tres principales puertos del Pacífico: Manzanillo y Lázaro Cárdenas, como los más importantes en cuanto a volumen de contenedores que manejan; y Ensenada, como el mejor ubicado en el corredor marítimo Este-Oeste del Hemisferio Norte.

El análisis AHP se enlaza con un modelo de Fortalezas, Oportunidades, Debilidades y Amenazas (FODA), con lo cual encontramos varias ventajas 
frente a la elaboración de números índices simplificados, el análisis exclusivamente cualitativo o la construcción de indicadores proxy de competitividad. Entre tales ventajas pueden mencionarse las siguientes: la combinación del análisis cualitativo, cuantitativo y gráfico riguroso, sin perder la perspectiva de los determinantes que más influyen en los indicadores centrales a valorar; la posibilidad de extender este análisis comparativo a un mayor número de puertos que se encuentran ubicados en un mismo ámbito de influencia; y el hecho de que su construcción puede sistematizarse de manera relativamente sencilla con programas informáticos accesibles, alimentados con información multidimensional que es relativamente fácil de elaborar.

\section{Fuentes de información}

La información analizada procede de tres fuentes. La primera se originó en entrevistas realizadas durante el periodo marzo-mayo de 2011 a personal adscrito a la Administración Portuaria Integral (API) de cada puerto. ${ }^{3}$ Se elaboraron seis entrevistas dirigidas a personal administrativo de las API, considerando a dos por puerto. Además, se entrevistó vía telefónica a seis usuarios destacados de los puertos, entre ellos a representantes de empresas navieras y comercializadoras en México, sumando un total de 12 entrevistas.

La segunda fuente se constituye por los Informes Estadísticos Mensuales de Movimientos de Carga, Buques y Pasajeros que publica la Coordinación General de Puertos y Marina Mercante de la Secretaría de Comunicaciones y Transportes. Finalmente, como tercera fuente se utilizaron los datos contenidos en los Programas Maestros de Desarrollo Portuario (PMDP) de Manzanillo, Lázaro Cárdenas y Ensenada. ${ }^{4}$

\footnotetext{
${ }^{3}$ Sociedad mercantil que mediante concesión para el uso, aprovechamiento y explotación de puertos, terminales e instalaciones, planea, programa, opera, desarrolla y administra los bienes y la prestación de los servicios.

${ }^{4}$ Ver Administración Portuaria Integral de Manzanillo (APIM, 2007), Administración Portuaria Integral de Ensenada (APIE, 2006) y Administración Portuaria Integral de Lázaro Cárdenas (APILC, 2011).
} 


\section{Determinantes de la competitividad portuaria}

Generalmente se identifica la competitividad de un puerto con su capacidad para captar, concentrar, manejar y distribuir contenedores a través de los océanos; por su capacidad para interconectarse con otros puertos, y destinos de acopio y distribución de bienes y mercancías; y por la amplitud de su radio de influencia local, regional o global tanto marítima como terrestre. Se considera también el impacto multiplicativo directo e indirecto que ejerce sobre otras actividades, que necesariamente deben desarrollarse tanto en los recintos portuarios como fuera de los mismos (García y Sánchez, 2006). Así, la capacidad portuaria depende no solamente de la infraestructura portuaria y tecnológica, sino también de las características geográficas del puerto, de su ubicación y grado de desarrollo de los diversos modos de transporte que le auxilian, así como de las estrategias comerciales que favorecen y orientan sus operaciones.

Uno de los elementos clave en la eficiencia y productividad de los puertos es su conectividad a través del intermodalismo marítimo y terrestre, de lo cual también depende la cobertura de servicios que provee en su zona de influencia. Requiriéndose para ello, una combinación eficiente del transporte ferroviario y carretero para el desplazamiento terrestre de la carga que alimenta al puerto o que es distribuida por éste. De allí que los insumos requeridos para la generación de un producto portuario competitivo - que en este caso podría ser la intensidad de las operaciones de carga-, habrán de considerar tanto indicadores tangibles - como es el área disponible, el número de contenedores desplazados por hora, y el tiempo ocioso de atraque - como cualidades estratégicas intangibles tales como: la localización geográfica y ámbito de influencia natural, y la capacidad de planeación, gestión y administración, por incidir estas últimas sobre el nivel de ingresos y los costos de operación.

Hasta mediados del siglo pasado, la ubicación geográfica era considerada como el factor principal que hacía posible el desarrollo de un puerto. La misma, determinaba la intensidad de intercambio de mercancías y bienes entre diferentes regiones, que por su cercanía relativa con otros puntos y centros de intercambio comercial, propiciaba que se movieran grandes cantidades de bienes de consumo que eran posteriormente distribuidos a grandes ciudades. 
Sin duda, la localización privilegiada favoreció que puertos crecieran y se desarrollaran como centros urbanos, propiciando que no sólo se constituyeran como centros de acopio y distribución sino también de demanda y consumo. Tal fue el caso de ciudades como Nueva York, Los Ángeles, Oakland, Seattle, Tacoma y Nueva Orleans en Estados Unidos; Southampton y Liverpool en Inglaterra; Rotterdam y Ámsterdam en Holanda; Bremen y Hamburgo en Alemania; Barcelona, Bilbao y Sevilla en España; Shanghai y Hong Kong en China; Singapur, Kobe y Yokohama en Japón; y Veracruz, Tampico, Manzanillo y Lázaro Cárdenas en México. Algunos de estos lugares han albergado por siglos puertos que han influido en la geopolítica del mundo y aún lo siguen haciendo, mientras que otros han emergido desde su condición de puertos locales a centros estratégicos para el desarrollo regional.

La dinámica y funcionalidad de un puerto se ve influida también a partir de la demanda de materia prima y bienes de consumo que, además de depender del crecimiento poblacional y económico, obligan a desarrollar una infraestructura entrelazada logísticamente que permita la conectividad portuaria para el acopio y distribución de las mercancías. En este sentido, la logística ha ido creciendo en complejidad a través del tiempo, de tal manera que las actividades de trasporte marítimo resultan cada vez más interdependientes de las llevadas a cabo por el transporte terrestre. Suelen conectarse ambas mediante nodos intermodales de alcance local, regional o continental, de los cuales depende en buena medida la calidad del servicio, los costos y la eficiencia portuaria.

Según Martner y Moreno (2004), la competitividad de los puertos a escala mundial se encuentra ligada a la continuidad entre las operaciones de primera y segunda maniobras. Al respecto, Bustos y colaboradores (2003) señalan que algunos puertos mexicanos han desarrollado infraestructura y recursos humanos para el desarrollo interno de operaciones de primera maniobra, lo cual les ha permitido optimizar la manipulación de la carga. Pero en la fase de segunda maniobra -integrada por las operaciones logísticas - el sistema no siempre funciona del todo bien. Esta pérdida de continuidad se debe a que no se han desarrollado mecanismos logísticos adecuados ni una conectividad suficiente con redes ferroviarias o de carreteras, traduciéndose esto en demoras, mermas y costos adicionales. 


\section{Planteamiento del Proceso Analítico Jerárquico para medir la competitividad}

La literatura no reporta un estricto listado de los aspectos a considerar para comparar y evaluar la competitividad. A escala internacional se han ensayado diversas metodologías para el caso del transporte marítimo. García y Sánchez (2006), por ejemplo, revisan la competitividad portuaria mediante un análisis comparativo de costos, calidad y capacidad de carga. Le-Griffin y Murphy (2006), por su parte, comparan la eficiencia de un puerto considerando como factores relevantes la capacidad de manejo de carga a partir de la intensidad en el uso de la infraestructura disponible en cada uno de ellos.

En el caso mexicano, Guerrero y Rivera (2009) aplican un modelo de análisis comparativo del desempeño de los puertos, con base en la medición de la productividad total de los factores. Para ello, definen a la productividad como el tonelaje manipulado que resulta de la combinación óptima de todos los insumos empleados en el proceso productivo, y como factores de producción, a los trabajadores y componentes de la infraestructura. Concluyen que los puertos de Progreso en Yucatán y Ensenada en Baja California son los que presentan las mayores potencialidades, aun cuando su flujo de contenedores es considerablemente menor al de los puertos de Manzanillo y Tampico.

Teniendo los anteriores estudios como referente, en este trabajo se valora la competitividad de los tres principales puertos mexicanos de la costa del océano Pacífico mediante el análisis AHP, y se analizan sus fortalezas, oportunidades, debilidades y amenazas en un contexto gráfico, que permite ubicar su posicionamiento en el análisis FODA a partir de valores relativos característicos. Como antecedente de este enfoque puede citarse a Chang y Huang (2006), quienes lo aplican para el caso de los principales puertos asiáticos.

El procedimiento AHP consiste en medir la competitividad a partir de varios criterios o determinantes complementarios, de los que se desprenden a su vez diversos subcriterios o factores que deben ser ponderados y jerarquizados para construir un índice. Para ello, el primer paso implica elaborar una matriz de comparación pareada entre los subcriterios considerados, siguiendo la metodología de valoración de Saaty (1990). 
Para mayor precisión y siguiendo a Hurtado (2002), considérese una matriz "A" cuadrada $n \times n$, que contiene comparaciones pareadas de alternativas o subcriterios. Sea además $\mathrm{a}_{\mathrm{ij}}$ el elemento $(i, j)$ de "A", para $i=1$, $2, \ldots n, \mathrm{y}, j=1,2, \ldots n$ subcriterios. Decimos entonces que "A" es una matriz de comparaciones pareadas de " $n$ " subcriterios o alternativas de medición de la competitividad portuaria, siendo $\mathrm{a}_{\mathrm{ij}}$ la medida de la preferencia de la alternativa en el renglón $i$ cuando se le compara con la alternativa de la columna $j$. Cuando $i=j$, el valor de $\mathrm{a}_{\mathrm{ij}}$ será igual a uno por estarse comparando un subcriterio consigo mismo.

De acuerdo con el axioma que refiere a la condición de juicios recíprocos, si "A" es una matriz de comparaciones pareada se cumple que: $\mathrm{a}_{\mathrm{ij}}=1 / \mathrm{a}_{\mathrm{ji}}$. Esto es, dicho elemento es equivalente al inverso que corresponde a los términos de $j$ e $i$ invertidos. Lo anterior puede plantearse de la siguiente forma matricial:

$$
\begin{aligned}
\mathrm{A} & =\left[\begin{array}{cccc}
1 & a_{12} & \ldots & a_{1 n} \\
a_{21} & 1 & \ldots & a_{2 n} \\
\vdots & \vdots & \vdots & \vdots \\
a_{n 1} & a_{n 2} & \ldots & 1
\end{array}\right] \\
\mathrm{A} & =\left[\begin{array}{cccc}
1 & a_{12} & \ldots & a_{1 n} \\
1 / a_{12} & 1 & \ldots & a_{2 n} \\
\vdots & \vdots & \vdots & \vdots \\
1 / a_{1 n} & 1 / a_{2 n} & \ldots & 1
\end{array}\right]
\end{aligned}
$$

Esto es, la especificación de la Matriz "A" en la forma indicada en (1) o (2) es equivalente. Una vez que se elabora esta matriz, se puede calcular lo que se denomina prioridad de cada uno de los elementos o subcriterios considerados para medir la competitividad. Para ello, el procedimiento seguido que conduce a la síntesis de los juicios de valor normalizados es el siguiente: 1) se suman los valores de cada columna de la matriz pareada; y, 2) se divide cada elemento por la suma de los elementos de la columna al que corresponde. Obteniéndose así, la matriz pareada normalizada.

La asignación de los valores ponderados para cada atributo, sigue la escala numérica sugerida por Saaty (2005), los cuales toman valores entre 
1 y 9 , reflejando los números impares mayor importancia. ${ }^{5}$ Las celdas alcanzan el valor de 1 cuando la comparación entre los subcriterios es igual de importante, y de 9 cuando uno de ellos es absolutamente más relevante que el otro. De manera que los números pares $(2,4,6$ y 8), representan valores intermedios entre dos juicios adyacentes.

Una vez definida la matriz pareada normalizada, se genera un vector de valores propios que refleja el orden de preferencias de los subcriterios. Para ello se procede de la siguiente forma: 1) se obtiene el cuadrado de la matriz pareada normalizada, esto es $A^{2} ; 2$ ) se obtiene un vector "E" de orden $n \times 1$, que resulta de la suma de los componentes de cada renglón de $\mathrm{A}^{2}$; 3) se normaliza el vector "E", dividiendo cada uno de sus componentes entre la sumatoria de todos ellos. De manera que el agregado total de sus elementos arroje la unidad; y, 4) los elementos del vector "E" normalizado, conforman los "n" valores propios preliminares.

El procedimiento anterior se repite, hasta encontrar una potencia " $n$ " a elevar la matriz pareada "A", que arroje valores propios que no resulten diferentes en más de dos décimas respecto al referente de la potencia anterior. Una vez identificada la matriz de interacción $A^{n}$, se obtienen los valores propios definitivos con base en el procedimiento antes expuesto. Para mayor detalle, ver Coyle (2004), Chang y Huang (2006) y Lee, Huang y Teng (2009).

Dado que se puede presentar cierto nivel de inconsistencia en las comparaciones pareadas que manifiesten los entrevistados, se aplica un método para controlarla mediante un contraste de estabilidad explicado por Alessio y Ashraf (2009), que implica el uso de una tasa de inconsistencia (RC) de la que se deriva un índice de inconsistencia aleatorio (IR), definido por Saaty (2005) como:

$$
I C=\frac{R C}{I R}
$$

${ }^{5}$ Por ejemplo, si el valor de A es absolutamente más importante que su par B, entonces el valor de la celda matricial sería 9. Si B es absolutamente menos importante que A, entonces toma el valor de 1/9 en la celda pareada. Ver Coyle (2004). 
Donde:

$$
\begin{aligned}
\mathrm{RC} & =\frac{\lambda_{\max -n}}{n-1} \\
\lambda_{\max } & =\frac{\sum_{j=1}^{n} a_{i j}}{a_{i n}} \\
\lambda_{\max } & =\text { Mayor autovalor de la matriz pareada de comparaciones } \\
\mathrm{n} & =\text { Número de criterios utilizados en la toma de decisión }
\end{aligned}
$$

Saaty demuestra que para valores de IC menores o iguales a 0.10 , la matriz pareada es relativamente consistente, mientras que si resultan mayores o iguales a 0.10 , podrá considerarse inconsistente. ${ }^{6}$

En suma, se implementan las siguientes técnicas para el presente análisis: a) definición de los criterios y subcriterios de medición de la competitividad portuaria; $b$ ) definición de la matriz de los factores determinantes; c) aplicación del proceso de análisis jerárquico; $d$ ) control del grado de inconsistencia de la matriz pareada de juicios de valor; y, $e$ ) adjudicación de la posición competitiva de los puertos en un diagrama esquemático FODA. Este procedimiento se llevó a cabo con el programa Expert Choice versión once. $^{7}$

\section{Determinantes y factores de la competitividad considerados}

Se considera como un reflejo general del grado de competitividad, el total de TEus $^{8}$ maniobrados en el año 2010 respecto a los insumos involucrados en cada uno de los puertos. Este es también un indicador de productividad

\footnotetext{
${ }^{6}$ Ver Saaty (1990).

${ }^{7}$ Ver: Expert Choice 11, en: http://expertchoice.com/

${ }^{8}$ TEU por sus siglas en inglés significa Twenty-foot Equivalent Unit. Es la unidad de medida de los contenedores de transporte de carga marítima, de 20 pies de largo, 8 de ancho, y 8.5 pies de altura.
} 
que refleja en cierta medida, la capacidad de un puerto para concentrar y distribuir bienes y servicios, como resultado de las acciones implementadas para evitar las amenazas y aprovechar las oportunidades, así como para minimizar las debilidades y apuntalar las fortalezas. Siguiendo esta idea, en la figura 2 se detallan en forma simplificada, algunos de los componentes del FODA portuario, según Lee et al. (2009) y Chang y Huang (2006).

Los criterios para la medición de la competitividad portuaria se clasifican en aquellos que tienen que ver con sus características físicas o infraestructurales, y los de naturaleza comercial. Los primeros incluyen el área total del puerto, el número de muelles con los que dispone, la capacidad portuaria en cuanto al número de Teus que movilizan, y la eficiencia de operación de carga, tal y como lo sugieren Martner y colaboradores (2010). Por su parte, las características comerciales que se retoman de Guerrero y Riviera (2009) tienen que ver con el número de navieras que operan en el puerto, los costos de los servicios principales y la conectividad con otros estados.

Figura 2. Distribución de los factores o subcriterios que determinan la competitividad en un puerto acorde con el modelo foda

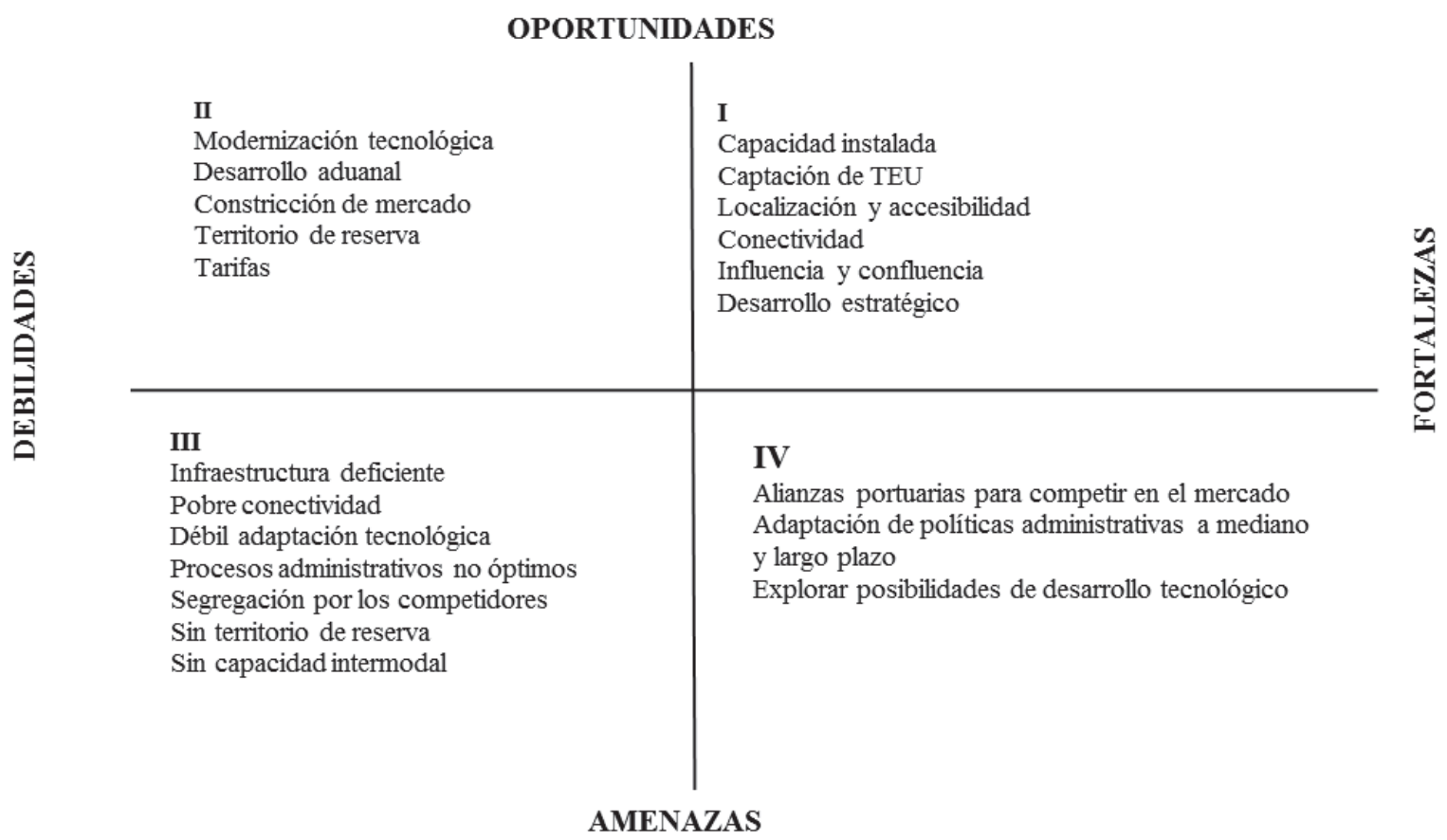

Fuente: Adaptado a partir de Lee et al. (2009) y Chang y Huang (2006). 
A partir de estos criterios, se desprendió un conjunto de subcriterios o factores (figura 3) con los cuales se obtuvieron mediciones diferenciadas de competitividad por puerto, considerando la importancia relativa ponderada que le asignaron los entrevistados a cada uno de esos factores. Con dicha información se construyeron dos matrices de comparación pareada de subcriterios - una para la de características físicas y otra para las de tipo comercial-, las cuales son requeridas por el Proceso Analítico Jerárquico y que reflejan la importancia ponderada de los factores, tanto para el caso de los que se valoraron en forma cualitativa en las entrevistas, como para los que fue posible asignarles un peso cuantitativo.

\section{Resultados}

En el cuadro 3 se presenta la información cuantitativa sobre las características físicas y comerciales de los puertos en estudio. Al respecto, los entrevistados

\section{Figura 3. Criterios y subcriterios considerados para la medición de la competitividad de los puertos}

\begin{tabular}{|c|c|c|}
\hline Puertos & $\begin{array}{l}\text { Determinantes de la } \\
\text { competitividad }\end{array}$ & Factores de la competitividad \\
\hline $\begin{array}{l}\text { Ensenada } \\
\text { Lázaro Cárdenas }\end{array}$ & $\begin{array}{l}\text { Características } \\
\text { físicas }\end{array}$ & $\begin{array}{l}\text { TEUs en } 2010 \\
\text { Número de muelles } \\
\text { Número de terminales } \\
\text { Terminales de contenedores } \\
\text { Área total } \\
\text { Área de contenedores } \\
\text { Eficiencia en la operación de carga }\end{array}$ \\
\hline Manzanillo & $\begin{array}{l}\text { Características } \\
\text { comerciales }\end{array}$ & $\begin{array}{l}\text { Costo puerto variable } \\
\text { Costo puerto fijo } \\
\text { Costo muellaje } \\
\text { Costo de atraque } \\
\text { Número de navieras } \\
\text { Hinterland (conexión con estados mexicanos) } \\
\text { Foreland (conexión con países) } \\
\text { Conectividad }\end{array}$ \\
\hline
\end{tabular}

Fuente: Elaboración propia con base en Saaty (2005) y Chang y Huang (2006). 
las valoraron en función del grado en que favorecen la atracción de flujos de contenedores a sus recintos. Además, mencionaron aquellos factores que promueven la competitividad mediante el impulso de negocios.

Con esta información se construyeron las matrices pareadas que se muestran en los cuadros 4 y 5 , mismas que describen en forma separada la ponderación relativa de las características físicas, por un lado, y las de naturaleza comercial, por otro. La última columna en ambas matrices representa la importancia relativa de cada factor, la cual se obtiene mediante la normalización de los valores relativos de la matriz y la obtención de los valores propios correspondientes.

\section{Cuadro 3. Criterios y subcriterios para la medición de la competitividad portuaria}

\begin{tabular}{|c|c|c|c|c|}
\hline \multirow[b]{2}{*}{ Tipo de característica/puertos } & \multirow[b]{2}{*}{ Unidades } & \multicolumn{3}{|c|}{ Puertos } \\
\hline & & Ensenada & $\begin{array}{c}\text { Lázaro } \\
\text { Cárdenas }\end{array}$ & Manzanillo \\
\hline \multicolumn{5}{|l|}{ Características físicas } \\
\hline Número de Muelles & & 2 & 19 & 29 \\
\hline Número de Grúas & & 8 & 12 & 38 \\
\hline Número de terminales & & 4 & 9 & 19 \\
\hline \begin{tabular}{|c|} 
Número de terminales \\
de contenedores
\end{tabular} & & 3 & 2 & 1 \\
\hline Área total & Hectáreas & 337 & 634 & 437 \\
\hline Área para contenedores & Hectáreas & 14.3 & 69.7 & 42.9 \\
\hline Total de contenedores en el 2010 & TEUS & 135606 & 796011 & 1509378 \\
\hline Eficiencia de operaciones & TEus/hora/grúa & 24 & 85 & 150 \\
\hline \multicolumn{5}{|l|}{ Características comerciales } \\
\hline Costo puerto variable & Pesos por unidad de arqueo & & & \\
\hline Costo puerto fijo & Pesos por embarque & 12320 & 19777 & 19777 \\
\hline Costo muellaje & Pesos por tonelada & 7.87 & 4.08 & 5.7 \\
\hline Atraque & Pesos por hora & 5.56 & 9.25 & 9.25 \\
\hline Número de Navieras & & 6 & 10 & 33 \\
\hline Hinterland & Número de Estados & 4 & 15 & 17 \\
\hline Foreland (Conexión con países) & Número de Países & 15 & 23 & 20 \\
\hline Conectividad * & & 1 & 3 & 3 \\
\hline
\end{tabular}

Fuente: Elaboración propia a partir de los programas maestros de desarrollo portuario. Ver APIE (2006, p. 6), APIM (2007) y APILC (2011). * $1=$ sólo acceso a carretera, $2=$ carretera y ferrocarril, y $3=$ carretera, ferrocarril y doble estiba. 
Cuadro 4. Matriz de comparaciones pareadas de las características físicas como primer criterio de la medición de la competitividad portuaria

\begin{tabular}{|c|c|c|c|c|c|c|c|c|c|}
\hline $\begin{array}{c}\text { Índice de } \\
\text { Consistencia: } 7.80 \%\end{array}$ & 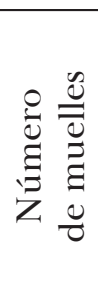 & 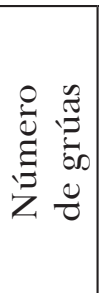 & 离 & 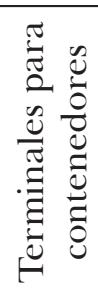 & 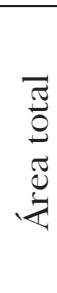 & 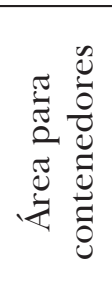 & 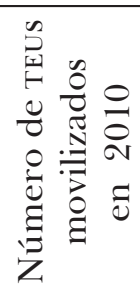 & 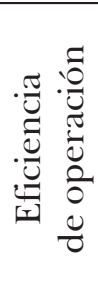 & 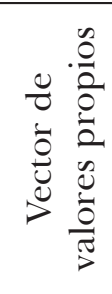 \\
\hline Número de muelles & 1 & $1 / 5$ & $1 / 5$ & $1 / 3$ & 3 & $1 / 5$ & $1 / 7$ & $1 / 3$ & $3.6 \%$ \\
\hline Número de grúas & 5 & 1 & 1 & 3 & 3 & 3 & 1 & 1 & $20.6 \%$ \\
\hline Núm. terminales & 5 & 1 & 1 & 5 & 7 & 1 & 1 & 3 & $20.9 \%$ \\
\hline $\begin{array}{l}\text { Terminales para } \\
\text { contenedores } \\
\end{array}$ & 3 & $1 / 3$ & $1 / 5$ & 1 & 5 & $1 / 5$ & $1 / 9$ & $1 / 3$ & $4.6 \%$ \\
\hline Área total & $1 / 3$ & $1 / 3$ & $1 / 7$ & $1 / 5$ & 1 & $1 / 5$ & $1 / 7$ & $1 / 3$ & $3.6 \%$ \\
\hline $\begin{array}{l}\text { Área para } \\
\text { contenedores }\end{array}$ & 5 & $1 / 3$ & 1 & 5 & 5 & 1 & 1 & 1 & $14.7 \%$ \\
\hline $\begin{array}{l}\text { Número de TEUs } \\
\text { movilizados en } 2010\end{array}$ & 7 & 1 & 1 & 9 & 7 & 1 & 1 & 1 & $18.4 \%$ \\
\hline Operación & 3 & 1 & $1 / 3$ & 3 & 3 & 1 & 1 & 1 & $13.6 \%$ \\
\hline
\end{tabular}

Fuente: Elaboración propia.

\section{Cuadro 5. Matriz de comparaciones pareadas de las características comerciales como segundo criterio de la medición de la competitividad portuaria}

\begin{tabular}{|c|c|c|c|c|c|c|c|c|c|}
\hline $\begin{array}{c}\text { Índice de } \\
\text { consistencia: } 8.5 \%\end{array}$ & 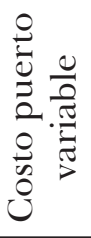 & 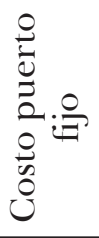 & 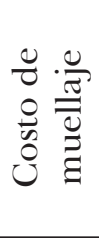 & 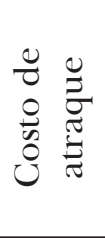 & 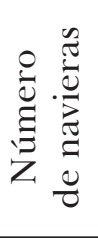 & 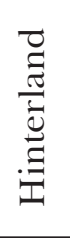 & 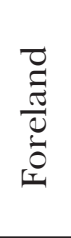 & 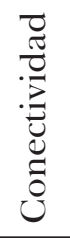 & 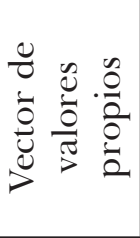 \\
\hline Costo puerto variable & 1 & 1 & 1 & 1 & 5 & $1 / 5$ & $1 / 5$ & $1 / 7$ & $5.0 \%$ \\
\hline Costo puerto fijo & 1 & 1 & 1 & 1 & $1 / 3$ & $1 / 5$ & $1 / 7$ & $1 / 5$ & $4.5 \%$ \\
\hline Muellaje & 1 & 1 & 1 & 1 & $1 / 5$ & $1 / 7$ & $1 / 5$ & $1 / 9$ & $3.9 \%$ \\
\hline Costo de atraque & 1 & 1 & 1 & 1 & 7 & $1 / 5$ & $1 / 3$ & $1 / 5$ & $6.4 \%$ \\
\hline Número de navieras & $1 / 5$ & 3 & 5 & $1 / 7$ & 1 & $1 / 7$ & $1 / 9$ & $1 / 9$ & $3.8 \%$ \\
\hline Hinterland & 5 & 5 & 7 & 5 & 7 & 1 & 1 & 1 & $25.3 \%$ \\
\hline Foreland & 5 & 7 & 5 & 3 & 9 & 1 & 1 & 1 & $24.9 \%$ \\
\hline Conectividad & 7 & 5 & 9 & 5 & 9 & 1 & 1 & 1 & $26.2 \%$ \\
\hline
\end{tabular}

Fuente: Elaboración propia. 
La matriz de jerarquización de las características físicas muestra que $60 \%$ de la importancia relativa de sus componentes está dado por el número de grúas, de terminales y de Teus manejados, con un peso individual promedio de 20\%; mientras que en el caso de las características comerciales, los componentes que presentan mayor importancia son la conectividad, el hinterland y el foreland con pesos individuales que rondan $25 \%$ y que, en conjunto, influyen en $76.4 \%$. Lo anterior resulta consistente con lo revisado en la literatura en cuanto a indicadores clave de competitividad portuaria, donde se acentúa tanto en la capacidad de manejo como en la conectividad y zona de influencia.

Cabe mencionar que los valores propios de las matrices contenidas en los cuadros 4 y 5 , se utilizaron para obtener el promedio ponderado para cada puerto, auxiliándose de las valoraciones asignadas por los entrevistados (cuadro 6). De estas últimas se obtienen los promedios o valor de referencia por puerto, con los que se generan las coordenadas características de la distribución portuaria en el análisis FODA (cuadro 7), consiguiéndose de esta forma la representación gráfica del posicionamiento competitivo de los puertos, dentro de los cuadrantes que se muestran en la figura 4.

Los resultados demuestran que el puerto de Ensenada se encuentra en el tercer cuadrante de las coordenadas FODA, evidenciando de esta manera un claro rezago competitivo en relación con el alcanzado por los dos mayores puertos del Pacífico. Las mayores desventajas de Ensenada en cuanto a características comerciales son: conectividad terrestre limitada, por no disponer de ferrocarril; un foreland de 15 países, cinco menos en relación con los otros dos puertos; un hinterland de sólo cuatro estados, en comparación con los 15 o más que alcanzan los mayores puertos del $\mathrm{Pa}$ cífico; y, aunque dispone de ventajas relativas en cuanto a costos de puerto fijo y atraque, aún no logra reducir sus altos costos de muellaje.

En el terreno de las características físicas, Ensenada se encuentra en desventaja en prácticamente todos los factores, destacando el hecho de que es el puerto con la menor área de contenedores, la cual representa la quinta parte de la del puerto Lázaro Cárdenas (ver cuadro 3).

El análisis jerárquico de las características comerciales indica que las áreas de influencia (hinterland y foreland) y la conectividad son los factores que observan la mayor contribución a la medición de esta dimensión 


\section{Cuadro 6. Valores medios ponderados de los subcriterios considerados para cada una de las características o criterios de medición de la competitividad portuaria}

\begin{tabular}{|c|c|c|c|c|}
\hline \multirow{2}{*}{$\begin{array}{l}\text { Tipo de característica / } \\
\text { valores por puerto }\end{array}$} & \multirow{2}{*}{$\begin{array}{l}\text { Valor } \\
\text { propio }\end{array}$} & \multicolumn{3}{|c|}{ Puertos } \\
\hline & & Ensenada & Lázaro Cádenas & Manzanillo \\
\hline \multicolumn{5}{|l|}{ Características físicas } \\
\hline Número de muelles & 0.04 & 0.07 & 0.65 & 1 \\
\hline Número de grúas & 0.21 & 0.21 & 0.32 & 1 \\
\hline Número total de terminales & 0.21 & 0.21 & 0.47 & 1 \\
\hline $\begin{array}{l}\text { Número de terminales } \\
\text { de contenedores }\end{array}$ & 0.05 & 0.5 & 1 & 0.5 \\
\hline Area total & 0.04 & 0.53 & 1 & 0.69 \\
\hline Area de contenedores & 0.15 & 0.2 & 1 & 0.61 \\
\hline $\begin{array}{l}\text { Contenedores movilizados } \\
\text { en TEUs , } 2010\end{array}$ & 0.18 & 0.09 & 0.53 & 1 \\
\hline Eficiencia de operación & 0.13 & 0.16 & 0.57 & 1 \\
\hline Promedio ponderado & 1 & 0.2 & 0.59 & 0.91 \\
\hline \multicolumn{5}{|l|}{ Características comerciales } \\
\hline Costo puerto variable & 0.05 & 1 & 0.38 & 0.38 \\
\hline Costo puerto fijo & 0.04 & 0.62 & 1 & 1 \\
\hline Costo muellaje & 0.04 & 1 & 0.52 & 0.72 \\
\hline Costo de atraque & 0.06 & 0.6 & 1 & 1 \\
\hline Número de navieras & 0.04 & 0.18 & 0.3 & 1 \\
\hline Hinterland (Estados mexicanos) & 0.25 & 0.23 & 0.88 & 1 \\
\hline Foreland (Conexión con países) & 0.25 & 0.65 & 1 & 0.87 \\
\hline Conectividad & 0.26 & 0.33 & 1 & 1 \\
\hline Promedio ponderado & 1 & 0.47 & 0.89 & 0.92 \\
\hline
\end{tabular}

Fuente: Elaboración propia, con base en entrevistas. 


\section{Cuadro 7. Valores característicos de la distribución foda para cada puerto}

\begin{tabular}{|l|cc|cc|}
\hline \multirow{2}{*}{ Puerto } & \multicolumn{2}{|c|}{ Características físicas } & \multicolumn{2}{c|}{ Características comerciales } \\
\cline { 2 - 5 } & $\begin{array}{c}\text { Promedio } \\
\text { ponderado }\end{array}$ & $\begin{array}{c}\text { Valor característico } \\
\text { del FODA en X }\end{array}$ & $\begin{array}{c}\text { Promedio } \\
\text { ponderado }\end{array}$ & $\begin{array}{c}\text { Valor característico } \\
\text { del FoDA en } Y\end{array}$ \\
\hline Ensenada & 0.204 & -0.364 & 0.473 & -0.29 \\
\hline Lázaro Cárdenas & 0.595 & 0.027 & 0.892 & 0.129 \\
\hline Manzanillo & 0.906 & 0.338 & 0.925 & 0.161 \\
\hline Valor Promedio & 0.568 & & 0.763 & \\
\hline
\end{tabular}

Fuente: Elaboración propia. Notas: el valor promedio ponderado por puerto se obtiene del cuadro 4, y los valores característicos resultan de la diferencia entre los valores promedios individuales y el valor promedio total.

Figura 4. Posicionamiento competitivo de los puertos en las coordenadas foda

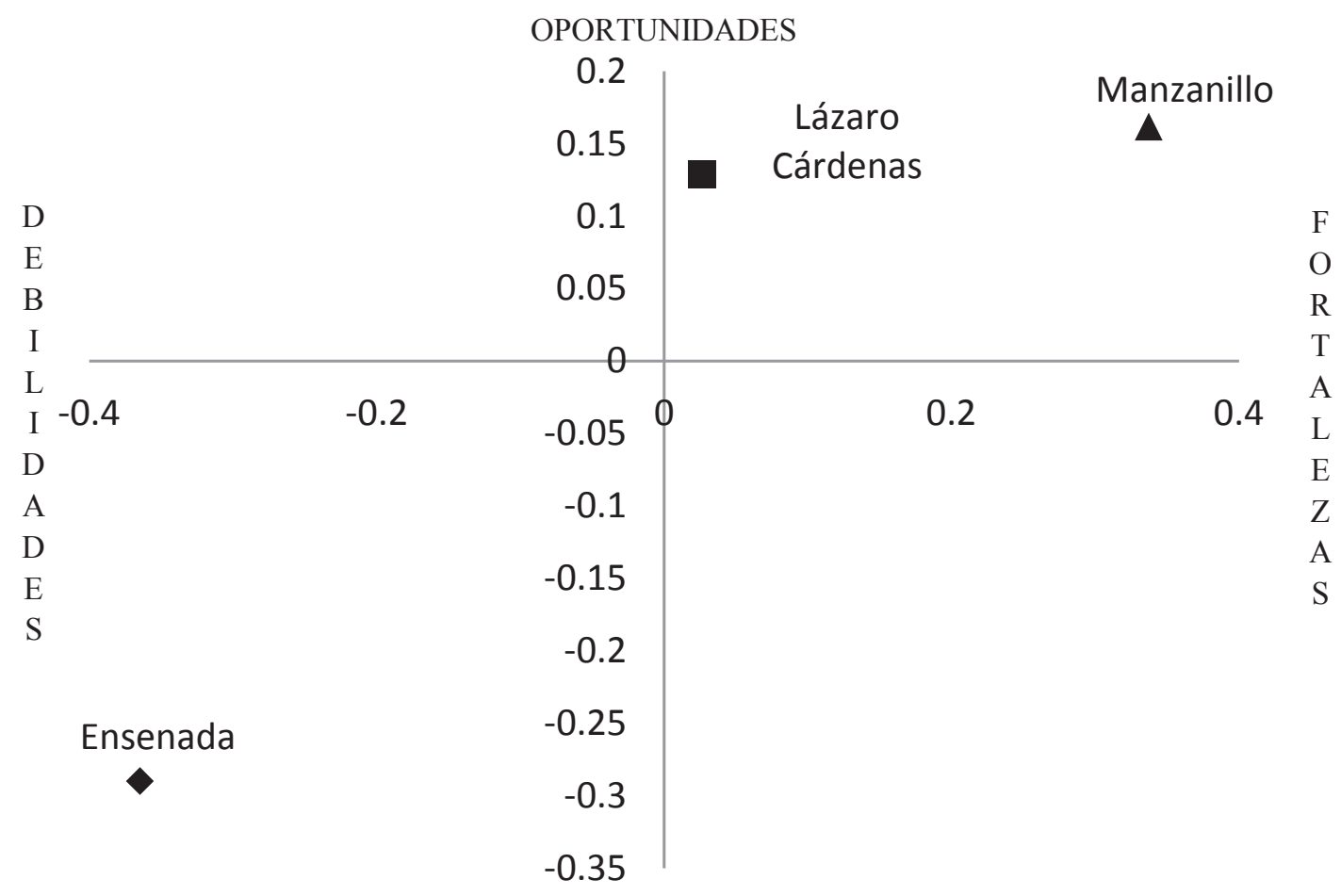

AMENAZAS

Fuente: Elaboración propia. 
de la competitividad, la cual asciende en forma conjunta a $73 \%$ de la misma (figura 5). En cuanto a las características físicas, se concluye que son el número de grúas y de terminales para contenedores los factores que más aportan a la competitividad infraestructural de los puertos analizados, contribuyendo en conjunto con $60 \%$ de la misma (figura 6).

El comportamiento por puerto de los determinantes de la competitividad es diferenciado. En la figura 5 se observa, por ejemplo, que los factores comerciales que mayor importancia revisten para el puerto de Manzanillo son el área de influencia nacional (hinterland) y la relación con un mayor número de compañías navieras; mientras que para el puerto de Lázaro Cárdenas son la conectividad junto con el área de influencia; y en cuanto al puerto de Ensenada, los precios en los rubros de puerto fijo y atraque representan los factores que más aportan al desempeño de la competitividad comercial, mientras que la conectividad y el área de influencia son los menos significativos.

Siguiendo el mismo orden de ideas, la figura 6 muestra que las características físicas con mayor relevancia para la competitividad infraestructural

\section{Figura 5. Comparativo del valor otorgado a las características} comerciales de los puertos y su contribución a la competitividad

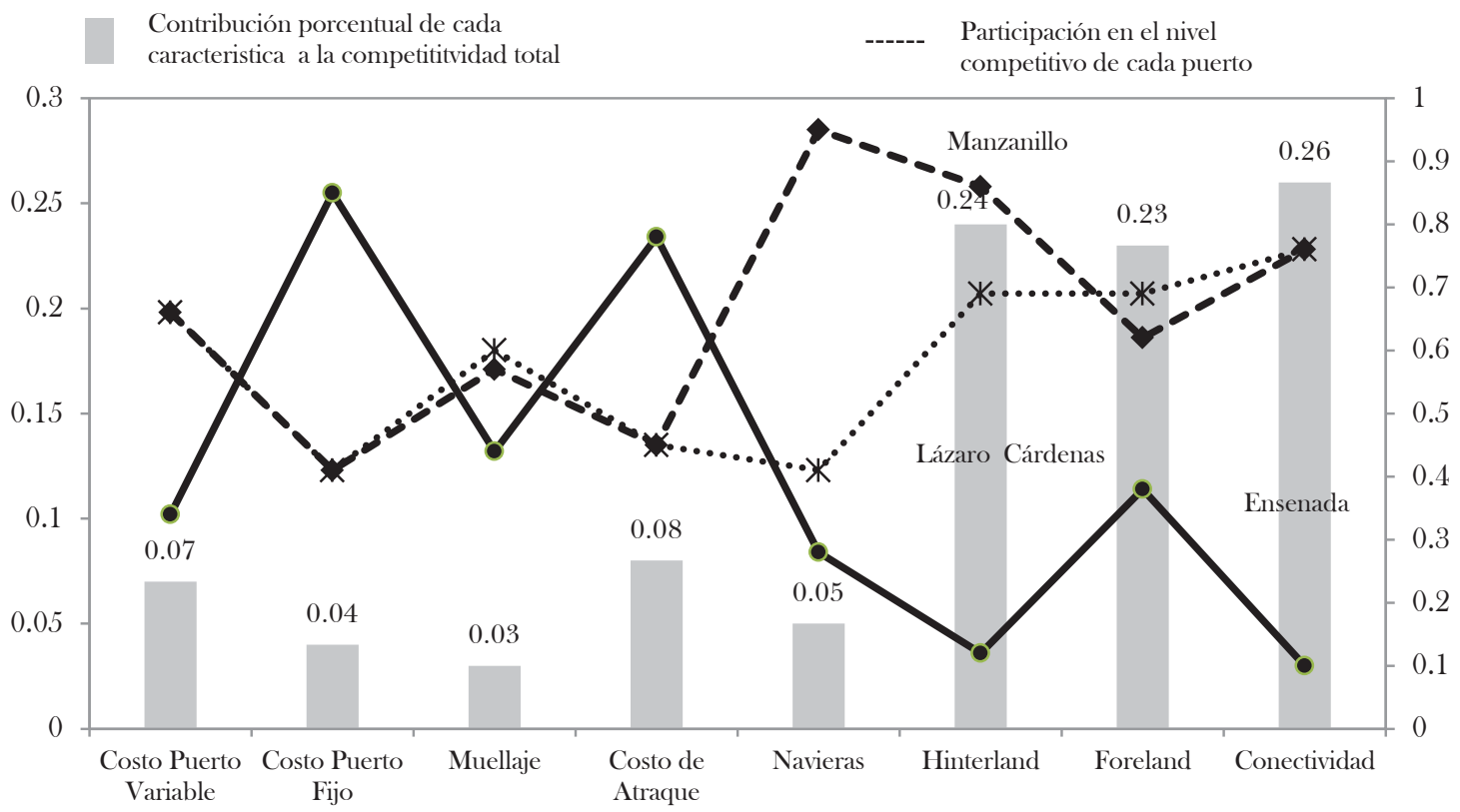

Fuente: Elaboración propia. 
Figura 6. Comparativo del valor otorgado a las características físicas de los puertos y su contribución a la competitividad

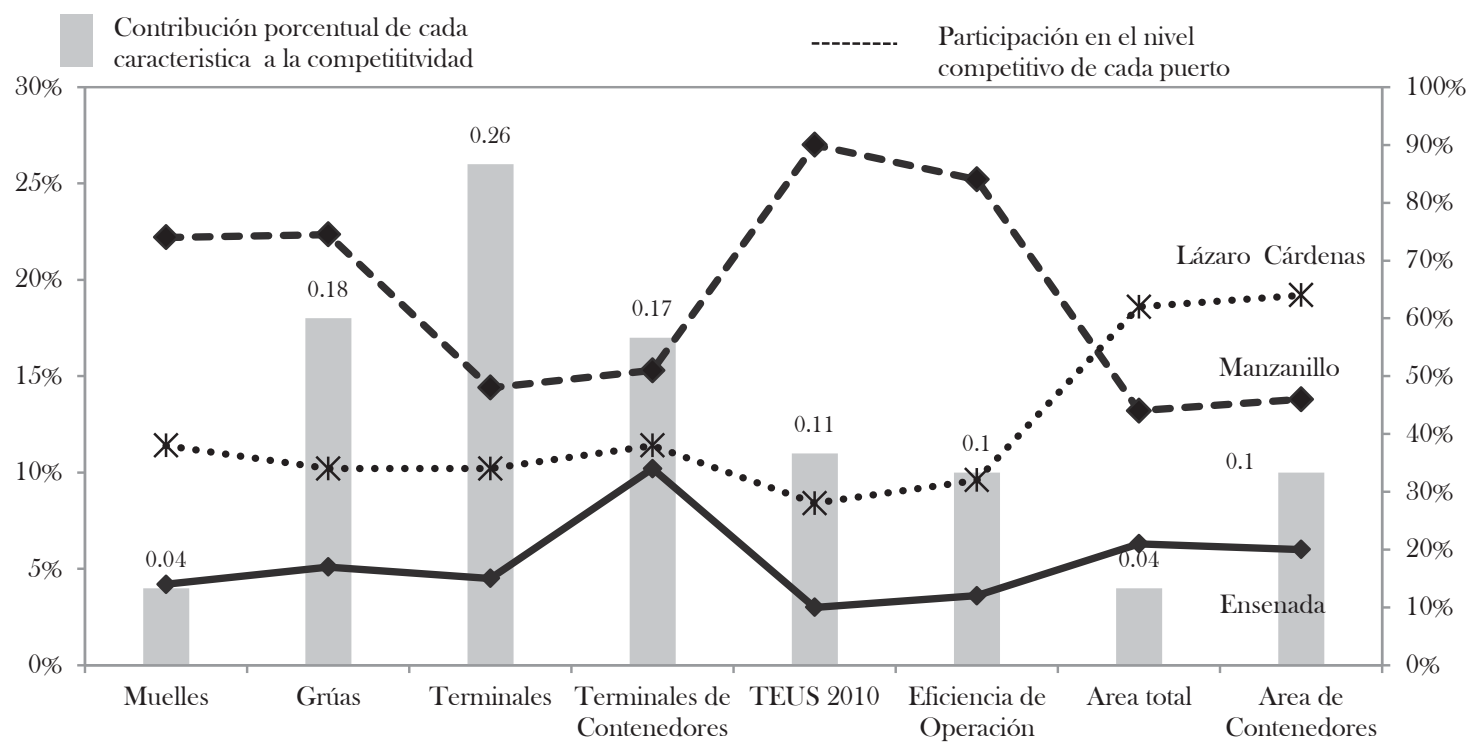

Fuente: Elaboración propia.

son, en el caso del puerto de Manzanillo, la superficie portuaria y de contenedores; mientras que para el de Lázaro Cárdenas son el número de TEus manipulados y la eficiencia en la operación de carga. Para el puerto de Ensenada, el número de grúas, de terminales y contenedores constituye los factores más influyentes en la competitividad infraestructural.

A diferencia del caso de Ensenada, la competencia por el movimiento de carga contenerizada entre los puertos de Manzanillo y Lázaro Cárdenas es más evidente, ya que su hinterland se traslapa al servir a varias entidades en común. ${ }^{9}$ Además, sus características competitivas son relativamente similares. En materia de factores comerciales para la competitividad, presentan indicadores parecidos, a excepción del número de navieras y al hecho de que Manzanillo posee un hinterland ligeramente más amplio. De acuerdo con la figura 6 -donde se establecen las aportaciones de cada factor infraestructural a la competitividad—, el puerto de Lázaro

${ }^{9}$ Entre ellos, Nuevo León, Tamaulipas, Querétaro, Distrito Federal, Estado de México y Guanajuato. Ver mapas de Martner (2010, p. 358). 
Cárdenas alcanza las mayores ventajas en cuanto a superficie de manejo, y desventajas en relación con el de Manzanillo respecto de la eficiencia en el manejo de operaciones y en la capacidad de carga, posicionándose este puerto como el que posee el mejor equipamiento e infraestructura. De allí que no sorprenda que sea el puerto que moviliza la mayor parte de la carga contenerizada del Pacífico.

\section{Conclusiones}

Se han identificado los determinantes comerciales y de infraestructura que influyen en el desempeño competitivo de los tres mayores puertos del Océano Pacífico mexicano, los cuales operan bajo el mismo régimen administrativo (la Administración Portuaria Integral) y, al menos los de Lázaro Cárdenas y Manzanillo, tienden a competir por zonas de influencia parcialmente compartidas. Dentro del análisis FODA se observa con claridad un claro rezago competitivo del puerto de Ensenada en relación con los otros dos puertos.

Si nos centramos en el caso del de Ensenada, encontramos que aunque su ubicación geográfica es un factor que favorece su participación en el mercado del manejo de contenedores, también debe tenerse presente que se encuentra localizado en un área que se considera altamente competitiva, donde se ubican puertos de gran capacidad como son los de Long Beach y Los Ángeles en California.

En este contexto, el puerto ensenadense ha desarrollado ventajas relativas en cuanto a costos de puerto fijo y atraque, pero no ha logrado abatir sus altos costos de muellaje. Además, se encuentra rezagado en materia de capacidad de operación, superficie y conectividad, lo que pudiera llegar a constituir una amenaza a su participación futura en el mercado de contenedores que, sin embargo, no necesariamente sería imputable a la presencia de los puertos de Manzanillo y Lázaro Cárdenas, pues la zona de influencia de los mismos es distinta a la de Ensenada.

De no mejorar su infraestructura, Ensenada podría perder oportunidades en el mercado de contenedores ante una eventual saturación de los puertos de Long Beach y Los Ángeles en Estados Unidos. Además de que podría desaprovechar parte de sus funciones como puerto de altura, de 
llegar a convertirse en un puerto alimentador o feeder, si todas las rutas troncales de Asia y América del Norte decidieran llegar solamente a los dos principales puertos del Pacífico, limitándose Ensenada a funciones de redistribución desde estos grandes puertos.

Con base en las recomendaciones de Chang y Huang (2006) para los puertos ubicados en el tercer cuadrante del diagrama FODA, como es el caso del puerto de Ensenada, resulta necesario que desarrollen estrategias enfocadas a la atención de mercados próximos y buques de cabotaje, para alternar como puerto secundario. De suerte que incluso si los puertos de Lázaro Cárdenas y Manzanillo logran consolidarse como hub regionales y/o amplían todavía más su vinculación con las rutas troncales de Asia y América del Norte, el de Ensenada podría resultar beneficiado como puerto feeder, sin que ello descarte que pueda aprovechar en mayor medida sus funciones de puerto de altura. Sobre esto último, podría especializarse en el manejo de carga mineral, pesquera, hortícola y de manufactura ligera, orientada al corredor marítimo Este-Oeste del hemisferio norte.

Por otro lado, la ubicación geográfica del puerto de Ensenada se puede considerar como estratégica ya que las distancias con los centros de población son relativamente cortas, ${ }^{10}$ lo cual puede influir favorablemente en una selección modal conveniente en términos de precios y costos (Bustos et al., 2003), como puede ser la conexión carretera y aeroportuaria. Esto es, aun cuando carece de conexión por ferrocarril, puede aprovechar sus conexiones carreteras y su cercanía con los centros de acopio y consumo más importantes del estado. Lo anterior le permitiría enfrentar mejor las oportunidades futuras, generando estrategias competitivas para atraer mercancías demandadas por los mercados de Tijuana, Mexicali, el sur de California y Arizona, de tal manera que aproveche nichos locales, regionales y transfronterizos que no se encuentran a una distancia mayor de 400 kilómetros del puerto.

Finalmente, consideramos que el proceso de análisis jerárquico (AHP) del que se desprenden los resultados aquí expuestos, puede ser aprovechado

${ }^{10}$ La distancia del puerto de Ensenada al de Los Ángeles es de 300 kilómetros; con respecto a Tijuana es de 111.5; y en relación con Mexicali 220 kilómetros; entre el puerto y Tecate median 106.5 kilómetros; y finalmente, en relación con Phoenix, Arizona, hay 457 kilómetros de distancia. 
para diseñar un observatorio de la competitividad, que incorpore mediante un monitoreo continuo en el tiempo, un mayor número de puertos nacionales y/o extranjeros. Siendo éste un ejemplo, de otros estudios que pudieran realizarse utilizando esta metodología.

\section{Bibliografía}

Administración Portuaria Integral de Ensenada (APIE). (2006). Programa Maestro de Desarrollo Portuario 2006-2011. Secretaría de Comunicaciones y Transportes, Ensenada, Baja California. Recuperado de < http://www.puertoensenada. com.mx/>.

Administración Portuaria Integral de Lázaro Cárdenas (APILC). (2011). Programa Maestro de Desarrollo Portuario Lázaro Cárdenas, 2011-2016. Secretaría de Comunicaciones y Transportes, Lázaro Cárdenas, Michoacán. Recuperado de <http://www.puertolazarocardenas.com.mx/>.

Administración Portuaria Integral de Manzanillo (APIM). (2007). Programa Maestro de Desarrollo Portuario del Puerto de Manzanillo 2007-2012. Secretaría de Comunicaciones y Transportes, Manzanillo, Colima. Recuperado de < http:// www.puertomanzanillo.com.mx $>$.

Alessio, I. y Ashraf, L. (2009). Analytic hierarchy process and expert choice: Benefits and limitations. OR Insight, 4(22), 201-220.

Bustos, A., Miranda, L. F., Acha, D. J., Aguerrebere, R., De la Torre, E. y Balbuena, J. A. (2003). Análisis del potencial para el intermodalismo en el movimiento de mercancías, proyecciones 2000, 2010, 2025. Sanfandila, Querétaro: Instituto Mexicano del Transporte.

Coyle, G. (2004). The Analytic Hierarchy Process (AHP). Pearson Education Limited, Open Acces Documents. Recuperado de <http://docs.google.com/>.

Chang, H. y Huang, W. (2006). Application of quantification swot analytical method. Mathematical and Computer Modeling, 43(1), 158-169.

García, A. L. y Sánchez, R. J. (Abril, 2006). Estadios de la competencia interportuaria: Del marco institucional a la conducta estratégica. VIII Reunión de Economía Mundial. Universidad de Alicante, España. Recuperado de <http://altea. daea.ua.es/ochorem/comunicaciones/MESA3COM/SanchezGarcia.pdf > .

Guerrero, A. y Rivera, C. (2009). México: Cambio en la productividad total de los principales puertos de contenedores. Revista CEPAL, 99, 175-187.

Hurtado, T. (2002). El Proceso de Análisis Jerárquico (AHP) como herramienta para la toma de decisiones. Manuscrito inédito, México.

Le-Griffin, H. D. y Murphy, M. (2006). Container terminal productivity: Experiences at the ports of Los Angeles and Long Beach. Estados Unidos: METRANS USC-CSUlb. 
Lee, Kuo-Liang, Wan, Wen-Chih y Teng, Junn-Yuan. (2009). Locating the competitive relation of global logistics hub using quantitative swoT analytical method. Qualtiy and Quantity, 1(43), 87-107.

Martner, C. D. y Moreno, A. (2004). Reestructuración portuaria e integración logística de los puertos mexicanos. Santiago de Querétaro, México: Instituto Mexicano del Transporte, Secretaría de Comunicaciones y Transporte.

Martner, C. D., Morales, C., Moreno, A., Pérez, A., Martínez, J. y De la Torre, E. (2010). Competencia y eficiencia de las cadenas de carga en México: Flujo de contenedores y gráneles agrícolas. Santiago de Querétaro, México: Secretaría de Comunicaciones y Transporte.

Martner, C. D. (2010). Puertos, espacio y globalización: El desarrollo de hub en México. Convergencia, 17(52), 319-360.

Saaty, T. L. (1990). How to make a decision: The Analytic Hierarchy Process. European Journal of Operational Research, 48(1), 9-26.

Saaty, T. L. (2005). Making and validating complex decisions with the AHP/ANP. Journal of Systems Science and Systems Engineering, 1(14), 1-36.

Secretaría de Comunicaciones y Transportes (SCT). (2001). Anuario Estadístico. México: Dirección General de Puertos.

Secretaría de Comunicaciones y Transportes (SCT). (2006). Anuario Estadístico. México: Dirección General de Puertos.

Secretaría de Comunicaciones y Transportes (SCT). (2008). Programa Nacional de Desarrollo Portuario 2007-2030. México: Puertos de México.

Secretaría de Comunicaciones y Transportes (SCT). (2009). Informe Estadístico Mensual: Movimiento de Carga, Buques y Pasajeros (Informe No. 52). México: Coordinación General de Puertos y Marina Mercante, Dirección General de Puertos.

Secretaría de Comunicaciones y Transportes (SCT). (2010). Estadísticas de los puertos de México, Anexo I: Rendimientos por tipo de carga. Enero-diciembre 2010 (No. 8). México: Dirección General de Puertos.

Secretaría de Comunicaciones y Transportes (sCT). (2013). Mapa de puertos de México. Dirección General de Puertos. Recuperado de la página de internet de la Secretaría de Comunicaciones y Transportes < http://www.sct.gob.mx/ index.php?id $=171>$.

Secretaría de Comunicaciones y Transportes (SCT). (2012). Anuario Estadístico. México: Dirección General de Puertos. 
